\title{
Coherently strained in-plane atomic layer heterojunctions
}

\author{
Chih-Kang Shih \\ NPG Asia Materials (2015) 7, e231; doi:10.1038/am.2015.127; published online 18 December 2015
}

Semiconductor heterojunctions (HJs) have played a critical role in many modern electronic and photonic devices. The emergence of transition metal dichalcogenides (TMDs) as a new class of twodimensional semiconducting materials creates exciting new opportunities to push semiconductor heterostructures toward a new frontier. Vertically stacked van der Waals heterostructures have been quickly recognized as a powerful platform to create atomically thin heterostructures. ${ }^{1}$ Recently, attention has been directed to the creation of monolayer 'lateral heterojunctions' with a line interface between two different TMDs, pushing semiconductor HJs to a new dimension. In late 2014, three papers published simultaneously, demonstrating direct growth of lateral heterojunctions using chemical vapor deposition. ${ }^{2-4}$ The papers demonstrated growth including both lattice matched and mismatched HJs; however, the interfaces were not chemically abrupt (that is, diffuse interface). In particular, the lattice mismatched system (for example, $\mathrm{WS}_{2}-\mathrm{WSe}_{2}$ ) displayed a compositional transition width of $40 \mathrm{~nm}$. Presumably, the gradual composition alleviates the abrupt change of the lattice constant, similar to the epitaxial growth of conventional heterostructures with a large lattice mismatch. Conventional wisdom suggests a chemically abrupt lateral interface in a lattice mismatched system would be difficult to achieve. In contrast, in 2015 by using a two-step process, Li et al. ${ }^{5}$ reported the successful growth of lattice-mismatched lateral $\mathrm{HJs}$ of $\mathrm{WSe}_{2}-\mathrm{MoS}_{2}$ with an atomically abrupt compositional interface as shown schematically in Figure 1a. This system is coherently strained judging by the high Photoluminescence efficiency of individual TMDs. This scenario is similar to the growth of coherently strained InAs quantum dots in GaAs, although in this case, compositional mixing at the interface has been difficult to eliminate. Thus, an atomically abrupt compositional interface in a system with such a large lattice mismatch comes as a pleasant surprise.

The strain variations in the $\mathrm{MoS}_{2}$ region grown via lateral epitaxy was measured using Raman spectroscopy. The photoluminescence peak energies also showed spatial variations consistent with the strain variations. Interestingly, both compressive and tensile strains are observed in the $\mathrm{MoS}_{2}$ region.

Department of Physics, University of Texas at Austin, Austin, TX, USA

E-mail: shih@physics.utexas.edu a

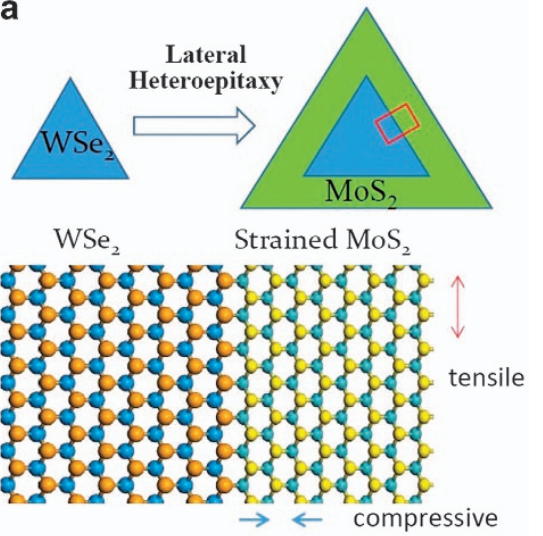

b

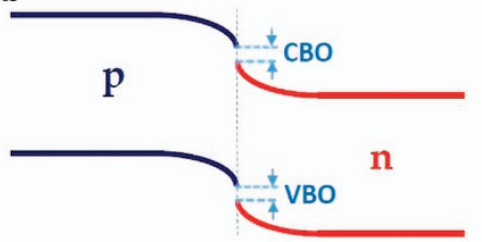

Figure 1 (a) Schematic showing the two step process of lateral heteroepitaxy: A single-layer $\mathrm{WSe}_{2}$ is first grown, followed by edge-nucleated lateral heteroepitaxy. The $\mathrm{MoS}_{2}$ is highly strained due to a large lattice mismatch. (b) A schematic showing the lateral band profile of the coherently strained in-plane atomic layer heterojunctions. Note that the band offset at the interface, as well as the band structure of individual TMD layer can be strongly influenced by the strain. The longrange band profile is determined by the dopant density and the carrier type.

One needs to recognize, however, these measurements are based on an optical technique whose resolution is diffraction limited. Moreover, the strain is inferred from the shift in Raman peaks assuming an isotropic strain. In reality, the local strain tensor is almost guaranteed to be anisotropic. Determination of the spatial variation of the strain tensor with finer spatial resolution (for example, nanometer scale) and their influence on local electronic structures will pave ways to tailor electronic structure via strain engineering.

As illustrated in Figure 1b, the lateral junction formed by the p-type $\mathrm{WSe}_{2}$ and n-type $\mathrm{MoS}_{2}$ also enabled them to demonstrate key device characteristics of a lateral $\mathrm{p}-\mathrm{n}$ junction (albeit not the homojunction) such as rectifications and photovoltaic responses, illustrating the potential for in-plane lateral junctions as building blocks for future two-dimensional electronic and photonic devices. Certainly much more research is required to bring these promises to reality. Many intriguing questions related to the growth mechanism of in-plane lateral heteroepitaxy remained to be answered. Moreover, how the anisotropic strain impacts the local electronic structure would need to be addressed. Finally, strategies need to be developed to engineer both type-I and type-II heterostructures.

\section{CONFLICT OF INTEREST}

The author declares no conflict of interest.

1 Geim, A. K. \& Grigorieva, I. V. Van der Waals heterostructures. Nature 499, 419-425 (2013).

2 Gong, Y., Lin, J., Wang, X., Shi, G., Lei, S., Lin, Z., Zou, X., Ye, G., Vajtai, R., Yakobson, B. I., Terrones, H., Terrones, M., Tay, B. K., Lou, J., Pantelides, S. T., Liu, Z., Zhou, W. \& Ajayan, P. M. Vertical and in-plane heterostructures from $\mathrm{WS}_{2} / \mathrm{MoS}_{2}$ monolayers. Nat. Mater. 13, 1135-1142 (2014).

3 Huang, C., Wu, S., Sanchez, A. M., Peters, J. J. P., Beanland, R., Ross, J. S., Rivera, P., Yao, W., Cobden, D. H. \& Xu, X. Lateral heterojunctions within monolayer $\mathrm{MoSe}_{2}-\mathrm{WSe}_{2}$ semiconductors. Nat. Mater. 13, 1096-1101 (2014).

4 Duan, X., Wang, C., Shaw, J. C., Cheng, R., Chen, Y., Li, H., Wu, X., Tang, Y., Zhang, Q., Pan, A., Jiang, J., Yu, R., Huang, Y. \& Duan, X. Lateral epitaxial growth of two-dimensional layered semiconductor heterojunctions. Nat. Nanotechnol. 9, 1024-1030 (2014).

5 Li, M.-Y., Shi, Y., Cheng, C.-C., Lu, L.-S., Lin, Y.-C., Tang, H.-L., Tsai, M.-L., Chu, C.-W., Wei, K.-H., He, J.-H., Chang, W.-H., Suenaga, K. \& Li, L.-J. Epitaxial growth of a monolayer $\mathrm{WSe}_{2}-\mathrm{MoS}_{2}$ lateral p$\mathrm{n}$ junction with an atomically sharp interface. Science 349, 524-528 (2015).

(c) (i) This work is licensed under a Creative Commons Attribution 4.0 International License. The images or other third party material in this article are included in the article's Creative Commons license, unless indicated otherwise in the credit line; if the material is not included under the Creative Commons license, users will need to obtain permission from the license holder to reproduce the material. To view a copy of this license, visit http://creativecommons.org/licenses/by/4.0/ 